\title{
Fairness and Efficiency in Online Advertising Mechanisms
}

\begin{abstract}
Alison Watts ${ }^{+}$
Department of Economics, Southern Illinois University, Carbondale, IL 62901, USA; wattsa@siu.edu

+ I would like to thank three anonymous referees whose comments and criticisms greatly improved the presentation and results.

Abstract: Online advertising often involves targeting ads to certain types of consumers where ads are commonly sold by generalized second price auctions. However, such an auction or mechanism could be considered unfair if similar consumers are consistently shown different ads or consistently receive different payoffs. Results show that such ascending bid auctions may result in unfair treatment and additionally that uncertainty regarding an ad's value can result in inefficiency. An alternative way to assign ads to consumers is presented called the random assignment mechanism. Results show that the random assignment can improve fairness while improving efficiency in some circumstances.
\end{abstract}

Keywords: auctions; online advertising; efficiency; fairness

JEL Classification: D44; L86; M3

\section{Introduction}

The fairness and efficiency of ascending bid auctions is examined in an online advertising framework where there is uncertainty regarding the ad's value to the consumer and where advertisers can target ads to certain consumers (Google has a remarketing campaign to help advertisers target previous visitors to their sites; see developers.google.com/adwords/api/docs/guides/remarketing for more information about remarketing. Such ads are sold using a bidding process similar to a generalized second price (gsp) auction).

Results give conditions under which ascending bid auctions are (and are not) fair and efficient in such a framework. Specifically, we show that the targeting of ads can lead to unfair treatment where similar consumers are treated differently. Additionally, uncertainty regarding an ad's value can lead to inefficiency if a consumer's interest level in an ad is quite different from what an advertiser expects.

In an effort to improve fairness and/or efficiency we also consider a random assignment mechanism where advertisers bid for a chance to display an ad and all advertisers above a certain threshold are given an equal chance to display an ad to any consumer (the idea of using randomization to address fairness is also used in the algorithmic classification literature of Dwork and Ilvento [1] and Dwork et al. [2]). By design, this mechanism does not allow an advertiser to target certain consumers. Results show that fairness is generally achieved by the random assignment mechanism in that similar consumers are shown or can expect to be shown similar ads. However, the random assignment mechanism has a similar inefficiency to the ascending bid auction due to uncertainty regarding the ad's value. Lastly, we also compare the a priori efficiency of the two mechanisms by comparing the probability that a mechanism assigns the efficient advertiser to show an ad given the uncertainty regarding ad values. Results show that the ascending bid auction is more likely to assign an efficient advertiser if there is an advertiser with a significantly higher common ad quality component than other advertisers. If the common quality components have little variation, then both mechanisms have a similar chance to assign the efficient advertiser. However, the random assignment mechanism may have an efficiency advantage in that it is less likely to assign the inefficient advertiser repeatedly. 
The current paper is most closely related to Athey and Ellison [3] and Jeziorski and Segal [4]. Athey and Ellison [3] consider sponsored link ad auctions where consumer search strategies are examined and show that a two-stage auction can increase efficiency in a model with click-weighting where interested consumers have similar ad values. Jeziorski and Segal [4] provides an empirical analysis of sponsored search ads with a theory component where ads have a consumer specific value component; Jeziorski and Segal [4] show that targeting ads can lead to a higher click through rate. Our analysis adds to this literature by considering the fairness of such auctions and shows that targeting consumers can lead to the unfair treatment of similar consumers. Additionally, we show that inefficiency can result even if consumers have a high click through rate if the value maximizing ad is not shown.

Other related literature inclues the growing literature on sponsored link auctions examing issues such as exclusive display (Sayedi et al. [5]), position ranking (Chu et al. [6]), and experimental studies of online auctions (Sun et al. [7]), as well as the classic generalized second price auction literature of Varian [8], Varian and Harris [9], and Edelman et al. [10]. There is also a literature examing online shopping with search costs (Choi et al. [11]) as well as consumer search for the best online price (Wang and Wright [12]). The targeting of ads has privacy issues regarding the disclosure of consumer information to advertisers; see De Corniere and De Nijs [13]. A literature review of privacy issues is presented by Acquisti et al. [14].

Additionally, our paper is related to the economics fairness literature of Rawls [15] and Roemer [16]; see also the literature reviews of Fleurbaey and Maniquet [17] and Roemer and Trannoy [18]. The current paper is also related to the fairness literature focusing on algorithmic fairness; see Dwork and Ilvento [1] and Dwork et al. [2]. See also Lepri et al. [19] for a literature review. Specifically, we define an ad display system to be fair if similar agents receive a similar payoff which is based on both Rawls [15]'s notion of egalitarian fairness and the multiple task fairness of Dwork et al. [2] and Dwork and Ilvento [1]. Additionally, we define an ad display system to be opportunity fair if similar agents have similar opportunities which is based on Roemer [16]'s notion of equality of opportunity. Our work differs from this previous work in that we apply these two fairness concepts to ascending bid auctions and the random assignment mechanism.

\section{Model}

There is a continuum of consumers who visit a website and observe $m$ displayed advertisements. Consumer $i$ can click on any of the ads displayed at a cost of $s_{i}$ per click. Assume that each $s_{i}$ is a random variable independently and identically distributed on $[0, \bar{s}]$ with a continuous distribution $H$. Additionally, assume $s_{i}$ is private information known only to $i$.

There are $n>m$ advertisers who want to advertise on the website. The value of clicking on advertiser $j$ 's ad to consumer $i$ is $v_{j i}=v_{j}+z_{j i}$, where $v_{j}$ represents a quality component common to everyone for $j$ 's ad and $z_{j i}$ represents a consumer specific quality component or interest level for $j$ 's ad. Assume that each $v_{j}$ and $z_{j i}$ are random variables independently and identically distributed from common distributions $F$ and $G$, respectively. Let $v_{j}$ and $z_{j i}$ have respective supports $[\underline{v}, \bar{v}]$ and $[\underline{z}, \bar{z}]$, where $\underline{v} \geq 0$ and $\underline{z} \geq 0$. Additionally, assume that initially each advertiser $j$ knows $v_{j}$, but only knows the distribution and support of $z_{j i}$. And initially each consumer $i$ only knows the distribution and support of $v_{j}$ and $z_{j i}$. However, if $i$ clicks on $j^{\prime}$ s ad then $i$ learns $v_{j i}$. As in Athey and Ellison [3], assume that if a consumer clicks on the ad, then the probability that the advertiser satisfies the consumer's need is $v_{j i}$, and if the need is met the advertiser receives a payoff of 1 . As $v_{j i}$ can be interpreted as a probability we assume that $\bar{v}+\bar{z} \leq 1$ and that $\underline{v}+\underline{z} \geq 0$.

Remark 1. Next we discuss the interpretation of $v_{j i}$ from both the consumer's and advertiser's perspective. When the consumer clicks on an ad they do not always purchase the product, but they do learn information or gain a perception about the product. In our model, this information or 
perception is of value $v_{j i}$ to the consumer and they learn this value after clicking, as in Jeziorski and Segal [4]. However, the advertiser only receives a payoff from the consumer if the consumer clicks on the ad and then purchases the product. We assume this purchase occurs with probability $v_{j i}$; we could have represented this probability as a function of $v_{j i}$ but chose to use $v_{j i}$ for simplicity. Thus, even though the consumer values the information at $v_{j i}$ they do not always purchase the product. See Zhang and Mao [20] and Chan and Park [21] for further discussion of the link between clicking on an ad and purchasing the product.

Let time be represented by $T=\{1,2, \ldots, t, \ldots\}$. Each period $t$ the advertisers are given the chance to bid on the $m$ displayed ads for each person $i$. We assume that consumers and advertisers remember what happened in previous periods. Thus, advertisers may use Bayesian updating to update their beliefs on $v_{j i}$ after observing whether or not a consumer clicks on their ad. While consumers who have previously clicked on an ad have learned $v_{j i}$ and know if it is worth clicking on again or not. For simplicity, consumers and advertisers are assumed to be myopic in that they are only trying to maximize current payoffs. Thus, advertisers do not try to maximize future payoffs.

We consider two different mechanisms for matching ads to consumers, the ascending bid auction and the random assignment mechanism. First, we define the ascending bid auction. As in a simplified version of Athey and Ellison [3] and Edelman et al. [10], the website runs an auction for the ads displayed to $i$. Let the auction start at zero and rise with a clock. Each advertiser then decides when to drop out. Let $j$ drop out of the auction at price $b_{j i}^{t}$. The auction ends when there is only one bidder left. Let the top $m$ bidders each display an ad to $i$ and let each pay the $m+1$ highest price per click. If there is no $(m+1)$ bid, then set this bid to 0 . Define $q_{j i}^{t}=1$ if $j$ is one of the top $m$ bidders to display an ad to $i$ and let $q_{j i}^{t}=0$ otherwise. We can interpret $q_{j i}^{t}$ as the probability that $j^{\prime}$ s ad is shown to $i$.

Note that here all bidders pay the $m+1$ highest price while in Athey and Ellison [3] and Edelman et al. [10] the $k$ th highest bidder receives the $k$ th ad spot and pays the $(k+1)$ highest price. In the current model, there is no advantage to having a higher ad spot as the consumer views all ad spots equally. Since our valuations are already complicated we chose not to further complicate them by having valuations or click through rates depend on display position. Thus, if winning advertisers paid different prices, then no advertiser would want to have the highest bid, but rather all advertisers would prefer to have the $m$ th highest bid. We avoid this scenario by having all winning advertisers pay the same price.

Notice that the ascending bid auction allows bidding for ads targeted at certain consumers such as those who have clicked on an ad previously or those with certain characteristics.

Second, we define the random assignment mechanism. Let each advertiser $j$ submit one bid $b_{j}^{t}$ for displaying an ad to any consumer in period $t$. Any advertiser who submits a bid above a floor of $\alpha$ is given an equal chance to display an ad to consumer $i$ at price $\alpha$ where bids are only paid if the ad is displayed. Here $\alpha$ is chosen from $[\alpha, \bar{\alpha}]$ by the website in period 1. Let $\hat{j}$ be the number of advertisers with $b_{j}^{t} \geq \alpha$. We define $q_{j i}^{t}=\frac{1}{j}$ for all $j$ with $b_{j}^{t} \geq \alpha$ and $q_{j i}^{t}=0$ otherwise. Thus, $q_{j i}^{t}$ represents the probability that $j^{\prime}$ s ad is shown to $i$.

Note that this mechanism does not allow an advertiser to make a bid to a specific consumer or type of consumer, but rather every period the advertiser makes one bid for display to any consumer. For $m>1$, we assume ads are displayed to $i$ in a random order.

In period $t$, let the ads displayed to $i$ be represented by $M_{i}^{t}$. Let the ads clicked on by $i$ be represented by $\tilde{M}_{i}^{t}$ with cardinality $\left|\tilde{M}_{i}^{t}\right|$. Then $i^{\prime}$ s period $t$ payoff is represented by $u_{i}^{t}=\sum_{j \in \tilde{M}_{i}^{t}} v_{j i}-\left|\tilde{M}_{i}^{t}\right| s_{i}$. And $i^{\prime}$ s payoff from clicking on $j^{\prime}$ s ad is represented by $u_{j i}^{t}=v_{j i}-s_{i}$.

We define an allocation of ads to consumers to be efficient if the sum of consumer payoffs are maximized. As each ad can be displayed to multiple consumers and a different set of ads can be displayed to each consumer the allocation is efficient if each consumer's payoff is maximized. Assume that for each $i$ there exists $m_{i}$ advertisers such that $v_{j i} \geq s_{i}$. Let an allocation, $M^{t}$, be efficient, if the ads displayed to each user maximize their payoff 
or if $M_{i}^{t}=\arg \max _{\hat{M} \in \mathcal{M}_{i}} \sum_{j \in \hat{M}} v_{j i}$ for all $i$. Here $\mathcal{M}_{i}$ represents all possible sets of ads of size $\min \left\{m, m_{i}\right\}$; thus, ads not worth clicking on for $i$ will never be efficient to display. Note that as only $\min \left\{m, m_{i}\right\}$ ads are shown to each $i, s_{i}$ is not needed in our maximization problem. Additionally, notice that since the advertiser's expected payoff from meeting a consumer's need is also equal to $v_{j i}$, our definition of efficiency also maximizes the sum of the expected payoffs to advertisers.

We define the ad display system to be fair, if similar agents receive a similar time $t$ payoff. Specifically, if there exists two agents $i$ and $\hat{i}$ such that $\left|v_{j i}-v_{j \hat{i}}\right|<\epsilon$ for all $j$ and $\left|s_{i}-s_{\hat{i}}\right|<\epsilon$ where $\epsilon$ is a small positive number. Then the ad display system is fair if $E\left[u_{i}^{t}\right]-E\left[u_{\hat{i}}^{t}\right]<m \cdot \epsilon$. This definition of fairness is based on the resource egalitarian fairness of Rawls [15], see also Fleurbaey and Maniquet [17]. Additionally, this definition of fairness is related to Dwork and Ilvento [1]'s notion of multiple task fairness which requires that similar individuals receive a similar expected outcome.

An alternative definition of fairness is that of opportunity fairness which states that similar agents should have similar opportunities. If $\left|v_{j i}-v_{j \hat{i}}\right|<\epsilon$ for all $j$ and $\left|s_{i}-s_{\hat{i}}\right|<\epsilon$ where $\epsilon$ is a small positive number, then the ad display system is opportunity fair if $\left|q_{j i}^{t}-q_{j i}^{t}\right|<\epsilon$. This fairness definition is related to Roemer [22]'s equality of opportunity; see also Roemer [23], and Roemer and Trannoy [18]. Under opportunity fairness, if agents have similar preferences and click costs, then each ad should have a similar chance of being displayed to them. Note that this definition allows agents to click on different ads while the previous fairness definition requires that agents also expect to click on the same set of ads.

Note that we analyze fairness in a setting with a homogeneous population. For an analysis of unfair advertising outcomes in a heterogeneous population, see Lambrecht and Tucker [24].

\section{Results}

We investigate the fairness and efficiency of the ascending bid auction and random assignment mechanism. First, consider the ascending bid auction.

Proposition 1. Let $v_{1}>v_{2}>\ldots>v_{n}$ and let $\underline{z}=\bar{z}$. Assume $v_{m}+\underline{z} \geq s_{i}$ for all $i$, where $v_{m}$ is the mth largest $v_{j}$. Then the ascending bid auction is efficient and opportunity fair for all $t$ and is fair at $t=1$.

If $\underline{z}=\bar{z}$, then all consumers have the same interest level for all ads and this interest level is known. Thus, the advertiser with the highest common quality component will also have the highest ad value for all consumers. The ascending bid auction will assign the advertisers with the $m$ highest common quality components to the consumer's $m$ ad slots. As the $j$ th ad has the $j$ th highest value, this allocation is efficient. This allocation is also opportunity fair as each consumer is shown the same set of ads.

Proof. As $\underline{z}=\bar{z}$, advertisers' initial beliefs regarding consumer valuations will be correct as all consumers will have the same interest level for all ads and this interest level is known. In the ascending bid auction, $j$ will be willing to bid up to $b_{j i}^{t}=v_{j}+\underline{z}$. The auction to display $m$ ads to $i$ will be won by the advertisers with the $m$ highest $v_{j}$ 's. As $v_{m}+\underline{z} \geq s_{i}$, it will always be beneficial for a consumer to click on one of these ads. There is no information for the advertiser to learn here regarding consumer preferences, so advertisers will not have an incentive to change their bids in the future. Advertisers $\{1,2, \ldots, m\}$ will be the only advertisers to display an ad. This auction is efficient since $\sum_{j=1}^{m}\left(v_{j}+\underline{z}\right)$ maximizes $i$ 's payoff or $\sum_{j=1}^{m}\left(v_{j}+\underline{z}\right)=\max _{\hat{M} \in \mathcal{M}_{i}} \sum_{j \in \hat{M}} v_{j i}$ for all $j$ and $i$. This auction is opportunity fair as all agents will have the same set of ads displayed to them.

This auction is fair if similar agents receive a similar expected payoff. Here all agents are displayed the same ads. We show that any two agents with similar click costs either 
both click on an ad, or both have expected utility within $\epsilon$. Consider $i$ and $\hat{i}$ such that $\left|s_{i}-s_{\hat{i}}\right|<\epsilon$. Without loss of generality assume $s_{i} \leq s_{\hat{i}}$. Assume $i$ clicks on a given ad or that $s_{i} \leq E\left[v_{k}\right]+\underline{z}$. If $s_{\hat{i}} \leq E\left[v_{k}\right]+\underline{z}$ then $\hat{i}$ also clicks on this ad. If instead $s_{\hat{i}}>E\left[v_{k}\right]+\underline{z}$, then $\hat{i}$ does not click on this ad. Consider the expected payoffs of $i$ and $\hat{i}$ in this case, when $t=1$. Here $E\left[u_{j i}^{1}\right]=E\left[v_{j}\right]+\underline{z}-s_{i}$ and $E\left[u_{j \hat{i}}^{1}\right]=0$. Thus, $E\left[u_{j i}^{1}\right]-E\left[u_{j \hat{i}}^{1}\right]=E\left[v_{j}\right]+\underline{z}-s_{i}$. Since $s_{i} \leq E\left[v_{k}\right]+\underline{z}<s_{\hat{i}}$ and $s_{\hat{i}}-s_{i}<\epsilon$ it must be that $E\left[v_{j}\right]+\underline{z}-s_{i}<\epsilon$. As each consumer is shown at most $m$ ads it must be that $E\left[u_{i}^{t}\right]-E\left[u_{\hat{i}}^{t}\right]<m \cdot \epsilon$. Thus, $i$ and $\hat{i}$ have similar expected payoffs and the auction is fair.

Proposition 1 assumes that $z_{j i}$ is the same for all consumers and that this is known to the advertisers. If however advertisers' beliefs regarding $z_{j i}$ are quite different from the actual $z_{j i}$, then the ascending bid auction may not be efficient or fair as the next set of propositions show.

Proposition 2. Let $m=1$. Let there exists advertisers $j$ and $k$ and consumer $i$ such that $v_{k}=$ $\max _{\ell} v_{\ell}>v_{j}$ and $z_{k i}-z_{j i}<v_{j}-v_{k}$. And assume $s_{i}<v_{k}+z_{k i}$ and $\max \left\{s_{i}, E\left[s_{i}\right]\right\}<E\left[v_{k}\right]+$ $E\left[z_{k i}\right]$. Then the ascending bid auction is not efficient.

In Proposition 2, advertiser $k$ has the largest common quality component and wins the ascending bid auction for consumer $i$. When $i$ is shown $k^{\prime}$ s ad the consumer learns it is worth clicking on and will continue to click on it in future periods. This act of clicking will reinforce $k^{\prime}$ s beliefs that $i$ values the ad and $k$ will continue to bid the most. However, consumer $i$ would receive a larger value from $j^{\prime}$ s ad as $z_{j i}$ is quite large but as $i$ is never shown $j$ 's ad the value of $z_{j i}$ is not learned. Thus, the ascending bid auction will result in an inefficient ad allocation.

Proof. At $t=1$, each advertiser $\ell$ will set $b_{\ell i}^{1}=v_{\ell}+E\left[z_{\ell i}\right]$. Advertiser $k$ will win the auction as $E\left[z_{\ell i}\right]$ is the same for all $\ell$ and as $v_{k}=\max _{\ell} v_{\ell}$. Since $s_{i}<E\left[v_{k}\right]+E\left[z_{k i}\right], i$ will click on $k^{\prime}$ s ad. As $s_{i}<v_{k}+z_{k i}$, after $i$ clicks on the ad they will learn that clicking on the ad was worth while as expected and will click on the ad again in future periods. From advertiser $k^{\prime}$ s perspective, $E\left[s_{i}\right]<E\left[v_{k}\right]+E\left[z_{k i}\right]$ so they expected $i$ to click on the ad and $i$ does. Advertiser $k^{\prime}$ s beliefs are confirmed as correct and they will not update their beliefs in period 1. In period $2, k$ will win the auction again and $i$ will click on the ad. Now $k$ will learn from $i$ 's click that $s_{i}<v_{k}+z_{k i}$ and $k$ will update $E\left[v_{k i}\right]$ upwards to $E\left[v_{k i} \mid s_{i}<v_{k}+z_{k i}\right]$. In future periods, $k$ will continue to win the auction and $i$ will continue to click.

As $k$ receives a payoff of 1 only if they meet $i$ 's need and this occurs with probability $v_{k i}$, it is possible with enough clicks $k$ will be close to learning $v_{k i}$. Regardless, $k$ continues to win the auction. However, since $z_{k i}-z_{j i}<v_{j}-v_{k}$ implies $v_{j}+z_{j i}>v_{k}+z_{k i}, i$ would be better off if $j$ won the auction. Thus, this auction is inefficient.

Remark 2. An interesting extension to the current framework would be to allow advertisers to occasionally experiment with their bids. In the proof of Proposition 2, advertiser $j$ has $v_{j i}>v_{k i}$ but never learns this information. If $j$ were allowed to experiment, then they might randomly submit the largest bid. Consumer $i$ would then click on the ad and would be willing to click on it again. In order for $j$ to learn that $i$ would click on their ad again, $j$ would need to experiment again and submit another large bid. Thus, $j$ might eventually learn that they should update their beliefs regarding $z_{j i}$. However, $k$ also learns to update their beliefs regarding $z_{k i}$. Thus, it is unlikely that $j$ would learn that $v_{j i}>v_{k i}$, but instead both $j$ and $k$ would update their beliefs upwards regarding $v_{j i}$ and $v_{k i}$. For simplicity, we do not allow this type of experimentation, but instead leave this for future research.

In the next proposition, we allow a new advertiser and consumer to enter the game at $t>1$ and show that such entry can affect fairness. Let $\hat{i}$ be a clone of $i$, if $s_{i}=s_{\hat{i}}$ and $z_{j i}=z_{j \hat{i}}$ for any $j$. 
Proposition 3. Let $m=1$. Let there exist advertiser $k$ and consumer $i$ such that $v_{k}=\max _{\ell} v_{\ell}$ and such that $s_{i}<v_{k}+z_{k i}$ and $E\left[s_{i}\right]<E\left[v_{k}\right]+E\left[z_{k i}\right]$. In period $t=3$, let a new advertiser $\hat{k}$ and a new consumer $\hat{i}$ enter the market where $v_{\hat{k}}=v_{k}+\beta$ and $\hat{i}$ is a clone of $i$. Then the ascending bid auction is neither opportunity fair nor fair in periods $t \geq 3$ for $\beta>0$ small enough.

Here $k$ wins the auction to display an ad to $i$ initially. As $i$ always clicks on $k$ 's ad, $k$ also learns that $i$ values the ad and will update upwards their expectation of $z_{k i}$. Thus, at $t=3, k$ will also update upwards their bid to display the ad to $i$. When the new advertiser $\hat{k}$ enters at $t=3$, they will win the auction to display the ad to $\hat{i}$ as $v_{\hat{k}}=v_{k}+\beta$. However, if $\beta$ is small enough, then $k$ will continue to win the auction to display to $i$. As $i$ and $\hat{i}$ are identical this auction allocation is neither fair nor opportunity fair since they will be shown different ads.

Proof. As $v_{k}=\max _{\ell} v_{\ell}>v_{j}$, advertiser $k$ will win the auction to display the ad to consumer $i$ in all periods. From the proof of Proposition 1, we know that after $i$ clicks on $k^{\prime}$ s link twice, $k$ will update their beliefs regarding $E\left[v_{k i}\right]$ from $E\left[v_{k i}\right]=v_{k}+E\left[z_{k i}\right]$ to $E\left[v_{k i} \mid s_{i}<v_{k}+z_{k i}\right]=$ $v_{k}+E\left[z_{k i} \mid s_{i}<v_{k}+z_{k i}\right]$. Next we show that $v_{k}+E\left[z_{k i}\right] \leq v_{k}+E\left[z_{k i} \mid s_{i}<v_{k}+z_{k i}\right]$ or that $E\left[z_{k i}\right] \leq E\left[z_{k i} \mid s_{i}<v_{k}+z_{k i}\right]$. If $\bar{s}<v_{k}+\underline{z}$, then it is always true that $s_{i}<v_{k}+z_{k i}$ and therefore $E\left[z_{k i}\right]=E\left[z_{k i} \mid s_{i}<v_{k}+z_{k i}\right]$. Now consider the case where $\bar{s} \geq v_{k}+\underline{z}$. After $i$ clicks on $k^{\prime}$ s link in period 2, $k$ learns that $s_{i}<v_{k}+z_{k i}$. For any given $s_{i}, k$ expects that $v_{k}+E\left[z_{k i}\right]>s_{i}$. Thus, if $s_{i}=v_{k}+\underline{z}+\epsilon$ then $k$ knows that $v_{k}+z_{k i} \notin\left[v_{k}+\underline{z}, v_{k}+\underline{z}+\epsilon\right]$, and $v_{k}+z_{k i}$ must instead be above this range. Similar analysis holds for larger $s_{i}$ resulting in $k^{\prime}$ s expectation of $z_{k i}$ increasing to $E\left[z_{k i} \mid s_{i}<v_{k}+z_{k i}\right] \geq E\left[z_{k i}\right]$.

In period 3, let a new advertiser, say $\hat{k}$, enter the market. Let this advertiser have $v_{\hat{k} i}$ with $v_{\hat{k}}=v_{k}+\beta$ where $0<\beta<E\left[z_{k i} \mid s_{i}<v_{k}+z_{k i}\right]-E\left[z_{k i}\right]$. Advertiser $k$ will still win the auction to display the ad to $i$ as the max $k$ is willing to bid is $v_{k}+E\left[z_{k i} \mid s_{i}<v_{k}+z_{k i}\right]$ which is greater than $v_{\hat{k}}+E\left[z_{\hat{k} i}\right]=v_{\hat{k}}+E\left[z_{k i}\right]$ which is the max $\hat{k}$ is willing to bid. Now let a clone of person $i$, say $\hat{i}$, enter the market in period 3 . Advertiser $\hat{k}$ will win the auction to display an ad for $\hat{i}$ as now what $k$ is willing to bid, $v_{k}+E\left[z_{k i}\right]$ is less than $v_{\hat{k}}+E\left[z_{\hat{k} \hat{i}}\right]=v_{\hat{k}}+E\left[z_{k i}\right]$ which is what $\hat{k}$ is willing to bid. Thus, $i$ and $\hat{i}$ will always view different ads and will receive different payoffs. Note that the payoffs $i$ and $\hat{i}$ expect are also different as $i$ expects to be shown $k^{\prime}$ s ad which they value at $v_{k i}$ and $\hat{i}$ is initially unsure which ad they will see, but will eventually expect to see $\hat{k}^{\prime}$ s ad with payoff $v_{\hat{k} i}$. Thus, the mechanism is not fair as the expected payoffs are different and is not opportunity fair as these agents expect to see different ads. In periods $t>3$ a similar result will occur. The only difference is that if with enough periods or clicks $k$ will be close to learning the true $z_{k i}$. However, their estimate should remain biased upwards, so for $\epsilon$ small enough the auction will continue to be both unfair and opportunity unfair for players $i$ and $\hat{i}$.

Remark 3. Note that in the proofs of Propositions 2 and 3 both the consumer and advertiser can update their beliefs. Consumer i learns $v_{j i}$ from clicking on an ad and then knows whether or not the ad is worth clicking on in the future. Advertiser $j$ observes whether $i$ clicks on their ad repeatedly and if $i$ does, then $j$ updates their beliefs regarding $z_{j i}$. An interesting extension to the current model would be to allow more uncertainty to $i$ 's updating. For instance, suppose $i$ does not learn $v_{j i}$ from clicking on the ad, but instead just learns more information regarding $v_{j i}$. Then $i$ 's updating would be more gradual and perhaps $i$ could even learn incorrect information regarding $v_{j i}$ which could cause $i$ not to click again on the ad even if they should. Such analysis would be different from the current model and we leave this analysis for future research.

Next we consider the random assignment mechanism of assigning advertisers to consumers to see if efficiency and fairness can be improved.

Proposition 4. The random assignment mechanism is opportunity fair for all $t$ and is fair at $t=1$. 
Opportunity fairness implies that similar agents expect to be shown the same set of ads while fairness implies that they also expect the same payoff. Even though similar agents expect to be shown the same ads, they may actually see different ads. Thus, similar agents can learn different information about which ads are worth clicking on. In later periods, similar agents may choose to click on different ads based on this information which will affect both future payoffs and future payoff expectations. Thus, fairness is guaranteed initially while opportunity fairness is always guaranteed.

Proof. First, we show that under the random assignment mechanism is opportunity fair or that similar individuals will expect to receive similar opportunities in all periods. Consider $i$ and $\hat{i}$ such that $\left|v_{j i}-v_{j i}\right|<\epsilon$ and $\left|s_{i}-s_{\hat{i}}\right|<\epsilon$. We show that such agents will expect to have the same ads displayed to them. Let advertisers $\{1,2, \ldots, \ldots, \bar{j}\}$ be the only advertisers with $b_{j}^{t} \geq \alpha$. Then each of these advertisers has an equal chance to display an ad to $i$ and $\hat{i}$. If $\bar{j} \geq m$, then $q_{j i}^{t}=q_{j \hat{i}}^{t}=\frac{m}{\bar{j}}$ for $j \in\{1,2, \ldots j, \ldots, \bar{j}\}$. If $\bar{j}<m$, then $q_{j i}^{t}=q_{j \hat{i}}^{t}=1$ for $j \in\{1,2, \ldots j, \ldots, \bar{j}\}$. And $q_{j i}^{t}=q_{j \hat{i}}^{t}=0$ for $j \in\{\bar{j}+1, \ldots, n\}$. Therefore, $i$ and $\hat{i}$ both expect to see the same ads and the random assignment mechanism is opportunity fair.

Second, we show that the mechanism is fair at $t=1$ or that initially $i$ and $\hat{i}$ expect to receive the same payoff. We already showed that each $j \in\{1,2, \ldots j, \ldots, \bar{j}\}$ has an equal chance of being shown to $i$ and $\hat{i}$. We next show that the expected payoff difference between $i$ and $\hat{i}$ for each such $j$ is less than $\epsilon$; which implies that $E\left[u_{i}^{t}\right]-E\left[u_{\hat{i}}^{t}\right]<m \cdot \epsilon$. Consider $j \in\{1,2, \ldots j, \ldots, \bar{j}\}$. If $j^{\prime}$ s ad is shown, then there are three possible actions by $i$ and $\hat{i}$. The first is that both $i$ and $\hat{i}$ click on $j^{\prime}$ s ad. In this case $i^{\prime}$ s expected payoff is $E\left[v_{j i}\right]-s_{i}$ and $\hat{i}^{\prime}$ s expected payoff is $E\left[v_{j i}\right]-s_{\hat{i}}$. As $E\left[v_{j i}\right]=E\left[v_{j \hat{i}}\right]$ it must be that $\left|E\left[v_{j i}\right]-s_{i}-E\left[v_{j i} \hat{i}\right]+s_{\hat{i}}\right|=\left|s_{\hat{i}}-s_{i}\right|<\epsilon$. The second case is that neither $i$ nor $\hat{i}$ click on $j$ 's ad in which case each consumer expects a payoff of 0 from the interaction. The third case is that one of the consumers clicks on the ad and the other does not. Without loss of generality, let $s_{i}<E\left[v_{j i}\right]=E\left[v_{\hat{j} i}\right]<s_{\hat{i}}$ and so $i$ clicks on $j^{\prime}$ s ad while $\hat{i}$ does not. Consumer $i$ 's expected payoff is $E\left[v_{j i}\right]-s_{i}$ and $\hat{i}^{\prime} \mathrm{s}$ expected payoff is 0 . The difference between the payoffs is $E\left[v_{j i}\right]-s_{i}$. Since $s_{i} \leq E\left[v_{j i}\right]<s_{\hat{i}}$ and $s_{\hat{i}}-s_{i}<\epsilon$ it must be that $E\left[v_{j i}\right]-s_{i}<\epsilon$. Thus, $i$ and $\hat{i}$ have similar expected payoffs and the auction is fair at $t=1$.

Let $v_{1}>v_{2}>\ldots>v_{n}$ and let $m$ be the advertiser with the $m$ th highest common quality component.

Proposition 5. Let $\underline{z}=\bar{z}$. The random assignment mechanism is efficient for all $t$, if $v_{m}+\underline{z} \geq$ $\alpha>v_{m+1}+\underline{z}$ and if $v_{m}+\underline{z} \geq s_{i}$ for all $i$.

If all consumers have the same interest level for all ads, then the random assignment mechanism is efficient if $\alpha$ is chosen so that only those advertisers with the top $m$ quality components are willing to submit a bid that is above this floor. These ads will be the only ones shown which is efficient.

Proof. As $\underline{z}=\bar{z}$, advertisers initial beliefs regarding consumer valuations will be correct. As $v_{m}+\underline{z} \geq \alpha>v_{m+1}+\underline{z}$ only advertisers $\{1,2, \ldots, m\}$ will submit bids high enough to participate in the auction, as $k$ will submit a bid of $v_{k}+\underline{z}$. This mechanism is efficient as it guarantees all consumers will be shown ads $\{1,2, \ldots, m\}$ and these are the ads that all consumer's value the most. Additionally, these ads are also worth clicking on for all $i$ since $v_{m}+\underline{z} \geq s_{i}$.

Note that if $\alpha \leq v_{m+1}+\underline{z}$, then more than $m$ advertisers will have bids greater than or equal to $\alpha$ and these advertisers will all have the same chance to display an ad to a consumer. Since each consumer values the top $m$ ads the most, this allocation will not always be efficient. If instead $\alpha>v_{m}+\underline{z}$, then advertiser $m$ will no longer submit a bid above $\alpha$ and so consumers will be shown less than the top $m$ ads which is also not efficient. 
Thus, if the search engine or website is concerned with efficiency here, then they would need to choose $\alpha$ so that only $m$ advertisers are willing to submit a bid. This would require some trial and error on the part of the website as they would simply need to raise $\alpha$ until only $m$ advertisers are willing to pay. It seems this solution would be desirable to the website as raising $\alpha$ in this way would most likely increase revenue. Nevertheless, resources would need to be devoted to selecting the correct $\alpha$ and such resources would not be used by the ascending bid auction. However, the ascending bid auction would be a much more complicated mechanism for the website to run as advertisers can select different bids for different consumers whereas with the random assignment each advertiser would only select one bid. Thus, it is unclear which mechanism dominates in turns of efficiency and/or ease of implementation when there is no heterogeneity in $z_{j i}$.

Continue to let $v_{1}>v_{2}>\ldots>v_{n}$.

Proposition 6. Let $m=1$ and let $z_{1 i}-z_{2 i}<v_{2}-v_{1}$. Assume $v_{1}+E\left[z_{1 i}\right]>\alpha>v_{2}+E\left[z_{2 i}\right]$ and $\max \left\{s_{i}, E\left[s_{i}\right]\right\}<v_{1}+z_{1 i}$ and $\max \left\{s_{i}, E\left[s_{i}\right]\right\}<v_{1}+E\left[z_{1 i}\right]$ for all $i$. Then the random assignment mechanism is not efficient.

Proof. As $s_{i}<v_{1}+z_{1 i}$ and $v_{1}+E\left[z_{1 i}\right]>\alpha>v_{2}+E\left[z_{2 i}\right]$, only advertiser 1 will submit a bid above $\alpha$ and 1's ad will be shown to all consumers. As $z_{1 i}-z_{2 i}<v_{2}-v_{1}$, consumer $i$ would be better off if 2's ad were shown. Thus, this allocation is not efficient. Note that as $\max \left\{s_{i}, E\left[s_{i}\right]\right\}<v_{1}+z_{1 i}$ and $\max \left\{s_{i}, E\left[s_{i}\right]\right\}<v_{1}+E\left[z_{1 i}\right]$ for all $i$, all consumers will click on 1's ad and this is also what 1 expects to have happen so 1 will not change their bid in future periods.

Note that the conditions of Proposition 6 are quite similar to that of Proposition 2. Thus, both mechanisms will assign the incorrect advertiser if the advertiser with the largest common quality component is not the advertiser with the largest value to the consumer.

In the next proposition we calculate the probability that the efficient advertiser is assigned to display an ad to $i$ by each mechanism. Recall that each $z_{j i}$ is randomly drawn from distribution $G$ and that advertisers do not initially have any additional information regarding $z_{j i}$.

Let $\tilde{n}$ represent the number of advertisers willing to bid in the random assignment mechanism or the number with $v_{j}+E\left[z_{j i}\right] \geq \alpha$.

Proposition 7. Let $m=1$ and $s_{i}=0$ for all $i$. Let $v_{k}=\max _{\ell} v_{\ell}$ and assume $v_{k} \geq \alpha-$ $\max \left\{z_{k i}, E\left[z_{k i}\right]\right\}$. Then the probability that the efficient advertiser is assigned to display an ad for consumer $i$ equals $\int_{\underline{z}}^{\bar{z}}\left(\prod_{j \neq k} G\left(\min \left\{v_{k}-v_{j}+z, \bar{z}\right\}\right) g(z) d z\right.$ under the ascending bid auction mechanism and the probability equals $1 / \tilde{n}$ under the random assignment mechanism.

For the random assignment mechanism, there are $\tilde{n}$ advertisers willing to bid above $\alpha$ and each will be given an equal chance to display an ad to $i$. Thus the probability that the efficient ad is shown to $i$ is $1 / \tilde{n}$. For the ascending bid auction, advertiser $k$ has the largest common component and will therefore win the auction to display the ad to $i$. However, if there exists advertiser $j$ with a larger value $v_{j i}>v_{k i}$, then efficiency will require $j$ to display the ad not $k$. The probability of this event occurring is given in the proposition.

Proof. First, consider the random assignment mechanism. Each advertiser $j$ will submit bid $b_{j}^{t}=v_{j}+E\left[z_{j i}\right]$ as this equals $j^{\prime}$ 's expected payoff of advertising to $i$. By assumption there are $\tilde{n}$ advertisers with $v_{j}+E\left[z_{j i}\right] \geq \alpha$. As the random assignment mechanism gives each advertiser who submits a bid above $\alpha$ an equal chance to display an ad, each advertiser with $b_{j}^{t} \geq \alpha$ has a chance of $1 / \tilde{n}$. As $v_{k}+z_{k i} \geq \alpha$ it must also be that $v_{\hat{k}}+z_{\hat{k} i} \geq v_{k}+z_{k i} \geq \alpha$ where $\hat{k}$ is the ad that maximizes $i$ 's payoff. Thus, the probability that the ad which maximizes $i$ 's payoff is displayed to $i$ is $1 / \tilde{n}$. Note that as $s_{i}=0$, all agents click on all ads thus, agents do not learn that some ads are less valuable than the clicking cost. Advertiser 
$j$ knows this and will have no incentive to update their bid. Thus, the probability that the correct ad is displayed is $1 / \tilde{n}$ for all $t$.

In the ascending bid auction, $k$ will win the auction to display an ad to $i$ as $v_{k}=$ $\max _{\ell} v_{\ell}$. Next we compute the probability that $k$ maximizes $i$ 's payoff and thus is the efficient advertiser to display an ad to $i$. Advertiser $k$ maximizes $i$ 's payoff if $v_{k i} \geq$ $v_{j i}$ for all $j \neq i$ or $v_{k}-v_{j} \geq z_{j i}-z_{k i}$. The probability that $v_{k}-v_{j} \geq z_{j i}-z_{k i}$ for a given $v_{k}-v_{j}$ equals $\int_{z}^{\bar{z}} G\left(\min \left\{v_{k}-v_{j}+z, \bar{z}\right\}\right) g(z) d z$. Using the cumulative distribution function of the largest order statistic, the probability that all $j \neq k$ have $v_{j i} \leq v_{k i}$ is $\int_{\underline{z}}^{\bar{z}}\left(\prod_{j \neq k} G\left(\min \left\{v_{k}-v_{j}+z, \bar{z}\right\}\right) g(z) d z\right.$.

Corollary 1. Let $m=1$ and $s_{i}=0$ for all $i$. Let $v_{k}=\max _{\ell} v_{\ell}$ and assume $v_{k} \geq \alpha-$ $\max \left\{z_{k i}, E\left[z_{k i}\right]\right\}$. Then the probability that the inefficient advertiser is assigned $\tau$ times in a row to display an ad for consumer i equals $\left(1-\int_{\underline{z}}^{\bar{z}}\left(\prod_{j \neq k} G\left(\min \left\{v_{k}-v_{j}+z, \bar{z}\right\}\right) g(z) d z\right)\right.$ under the ascending bid auction mechanism and the probability equals $(1-1 / \tilde{n})^{\tau}$ under the random assignment mechanism.

This corollary follows directly from Proposition 7 as the random assignment mechanism has a $(1-1 / \tilde{n})$ chance of assigning the inefficient ad each period while the ascending bid auction has a $1-\left(\int_{z}^{\bar{z}}\left(\prod_{j \neq k} G\left(\min \left\{v_{k}-v_{j}+z, \bar{z}\right\}\right) g(z) d z\right)\right.$ of assigning the inefficient ad the first period. Here ad $k$ is always assigned and it is inefficient with the probability given. In subsequent periods, $k$ will continue to win the auction, thus given that the inefficient ad is initially assigned, the inefficient ad will be assigned in future periods with probability 1 .

In the following example we compare the probability of assigning the efficient advertiser by the random assignment mechanism to that of the ascending bid auction. The example illustrates that the ascending bid auction is more likely to choose the efficient advertiser if there is an advertiser whose common quality component is significantly larger than the other common components as this advertiser is likely to be efficient and will always be chosen by the ascending bid auction. If the common quality components have little variation, then the ascending bid auction and the random assignment mechanism have a similar probability of choosing the efficient advertiser. Additionally, we show that the random assignment mechanism may have an efficiency advantage over time in that it is less likely that a consumer is shown an inefficient ad repeatedly with the random assignment mechanism.

Example 1. Let $z_{j i} \sim U[0,0.5], n=3, m=1,\left(v_{1}, v_{2}, v_{3}\right)=(0.4,0.35,0.35), \alpha=0.3$, and let $s_{i}=0$ for all $i$. First, consider the ascending bid auction. As advertiser 1 has the largest $v_{j}, 1$ will have the highest bid and will win the auction to display the ad to $i$. However, it will only be efficient for 1 to win the auction if $v_{1 i}>v_{2 i}$ and $v_{1 i}>v_{3 i}$. From Proposition 7 , the probability that $v_{1 i}>v_{2 i}$ given $v_{1}-v_{2}=0.05$ equals $\operatorname{Pr}\left(z_{2 i}-z_{1 i}<0.05\right)=\int_{0}^{0.45} \frac{0.05+z}{0.5} \cdot \frac{1}{0.5} d z+\frac{0.05}{0.5}=0.59$. The probability that $z_{2 i}-z_{1 i}<0.05$ and $z_{3 i}-z_{1 i}<0.05$ equals $\int_{0}^{0.5}\left(\frac{\min \{0.05+z, 0.5\}}{0.5}\right)^{2} \cdot \frac{1}{0.5} d z=0.43$. Here even though advertiser 1 has the largest $v_{j}$, they may not have the largest $v_{j i}$ and thus may not be the efficient ad choice. Now we compare this probability to that of the random assignment mechanism. By Proposition 7, as all advertisers have $v_{j}+E\left[z_{j i}\right]=v_{j}+0.25 \geq \alpha=0.3$ the probability that the efficient advertiser is assigned is $\frac{1}{3}<0.43$. Notice that the ascending bid auction has a higher probability of assigning the efficient advertiser than does the random assignment mechanism. Here ad 1 has the highest quality component and is thus likely to have the largest value; additionally, ad 1 is always chosen with the ascending bid auction.

We also investigate what happens to these probabilities as the gap between the quality compo$n e n t s$ decreases. Here we consider the same example, but now let $\left(v_{1}, v_{2}, v_{3}\right)=(0.4,0.39,0.39)$. In the random assignment mechanism, the probability that the efficient advertiser is selected remains at $\frac{1}{3}$. In the ascending bid auction, the probability that the efficient advertiser is selected is now equal 
to $\int_{0}^{0.5}\left(\frac{\min \{0.01+z, 0.5\}}{0.5}\right)^{2} \cdot \frac{1}{0.5} d z=0.35$. Thus, as the difference between the quality components decreases, the two mechanisms have a similar probability of choosing the efficient allocation.

Next we investigate the probability that an inefficient allocation is made three periods in a row when $\left(v_{1}, v_{2}, v_{3}\right)=(0.4,0.35,0.35)$. In the random assignment mechanism, there is a $\left(1-\frac{1}{3}\right)=\frac{2}{3}$ chance each period of assigning an inefficient advertiser. Thus, the probability that an inefficient allocation is chosen three times in a row is $\left(\frac{2}{3}\right)^{3}=0.29$. In the ascending bid auction, there is a $1-0.43=0.57$ chance that the inefficient allocation occurs in period 1 or that advertiser 1 is not the efficient advertiser. In period 2, advertiser 1 is still chosen and thus the probability that the inefficient allocation is made in period 2 given that the inefficient decision was made in period 1 equals 1. Similarly, the probability that the inefficient decision is made three times in a row remains at 0.57 . Thus, the random assignment mechanism has an advantage in that consumers are more likely to eventually view the efficient ad than they are with the ascending bid auction.

\section{Conclusions}

The fairness and efficiency of ascending bid auctions and a random assignment mechanism are examined in an online advertising framework. Results show that the random assignment mechanism can increase fairness while the ascending bid auction may increase efficiency. However, the random assignment mechanism may decrease the chance that an inefficient ad is shown repeatedly.

Possible extensions include examining group fairness. Our fairness notions ask that similar people have similar opportunities or similar payoffs. Group fairness would ask that groups of similar people are treated equally; see Dwork and Ilvento [1], Dwork et al. [2]. In our framework, one might ask that a group of demographically similar people have an equal chance to see a valuable ad as another group. One could then compare the ascending bid auction and the random assignment mechanism in terms of group fairness. It seems likely that the random assignment mechanism would treat the demographic groups similarly, but that the ascending bid auction may not. One could then investigate if there are conditions on ad values under which the ascending bid auction would treat the groups similarly. Such an approach would differ from that of Dwork and Ilvento [1] and Dwork et al. [2] who investigate the individual and group fairness of algorithms and show that algorithms which satisfy such fairness in isolation may not in a system. Our approach would add to this literature by focusing on the group fairness of two specific mechanisms for ad assignment namely the ascending bid auction and the random assignment mechanism. We leave such an analysis for future research.

Funding: This research received no external funding.

Institutional Review Board Statement: Not applicable.

Informed Consent Statement: Not applicable.

Data Availability Statement: Not Applicable.

Conflicts of Interest: The author declares no conflict of interest.

\section{References}

1. Dwork, C.; Ilvento, C. Fairness under composition. arXiv 2018, arXiv:1806.06122.

2. Dwork, C.; Hardt, M.; Pitassi, T.; Reingold, O.; Zemel, R. Fairness through awareness. In Proceedings of the 3rd Innovations in Theoretical Computer Science Conference, Cambridge, MA, USA, 8-10 January 2012.

3. Athey, S.; Ellison, G. Position auctions with consumer search. Q. J. Econ. 2011, 126, 1213-1270. [CrossRef]

4. Jeziorski, P.; Segal, I. What makes them click: Empirical analysis of consumer demand for search advertising. Am. Econ. J. Microecon. 2015, 7, 24-53. [CrossRef]

5. Sayedi, A.; Jerath, K.; Baghaie, M. Exclusive placement in online advertising. Mark. Sci. 2018, 37, 970-986. [CrossRef]

6. Chu, L.Y.; Nazerzadeh, H.; Zhang, H. Position ranking and auctions for online marketplaces. Manag. Sci. 2020, 66, 3617-3634. [CrossRef]

7. Sun, H.; Fan, M.; Tan, Y. An empirical analysis of seller advertising strategies in an online marketplace. Inf. Syst. Res. 2020, 31, 37-56. [CrossRef]

8. Varian, H.R. Position auctions. Int. J. Ind. Organ. 2007, 25, 1163-1178. [CrossRef] 
9. Varian, H.R.; Harris, C. The vcg auction in theory and practice. Am. Econ. Rev. 2014, 104, 442-445. [CrossRef]

10. Edelman, B.; Ostrovsky, M.; Schwarz, M. Internet advertising and the generalized second-price auction: Selling billions of dollars worth of keywords. Am. Econ. Rev. 2007, 97, 242-259. [CrossRef]

11. Choi, M.; Dai, A.Y.; Kim, K. Consumer search and price competition. Econometrica 2018, 86, 1257-1281. [CrossRef]

12. Wang, C.; Wright, J. Search platforms: Showrooming and price parity clauses. Rand J. Econ. 2020, 51, 32-58. [CrossRef]

13. De Corniere, A.; De Nijs, R. Online advertising and privacy. Rand J. Econ. 2016, 47, 48-72. [CrossRef]

14. Acquisti, A.; Taylor, C.; Wagman, L. The economics of privacy. J. Econ. Lit. 2016, 54, 442-492. [CrossRef]

15. Rawls, J. A Theory of Justice; Harvard University Press: Cambridge, MA, USA, 1971.

16. Roemer, J.E. Equality of resources implies equality of welfare. Q. J. Econ. 1986, 101, 751-784. [CrossRef]

17. Fleurbaey, M.; Maniquet, F. Optimal income taxation theory and principles of fairness. J. Econ. Lit. 2018, 56, 1029-1079. [CrossRef]

18. Roemer, J.E.; Trannoy, A. Equality of opportunity: Theory and measurement. J. Econ. Lit. 2016, 54, 1288-1332. [CrossRef]

19. Lepri, B.; Oliver, N.; Letouzé, E.; Pentland, A.; Vinck, P. Fair, transparent, and accountable algorithmic decision-making processes. Philos. Technol. 2018, 31, 611-627. [CrossRef]

20. Zhang, J.; Mao, E. From online motivations to ad clicks and to behavioral intentions: An empirical study of consumer response to social media advertising. Psychol. Mark. 2016, 33, 155-164. [CrossRef]

21. Chan, T.Y.; Park, Y.H. Consumer search activities and the value of ad positions in sponsored search advertising. Mark. Sci. 2015, 34, 606-623. [CrossRef]

22. Roemer, J.E. A pragmatic theory of responsibility for the egalitarian planner. Philos. Public Aff. 1993, 22, $146-166$.

23. Roemer, J.E. Equality of Opportunity; Elsevier: Amsterdam, The Netherlands, 1998.

24. Lambrecht, A.; Tucker, C. Algorithmic bias? an empirical study of apparent gender-based discrimination in the display of stem career ads. Manag. Sci. 2019, 65, 2966-2981. [CrossRef] 